\title{
A study of PTEN expression in endometrial hyperplasia and endometrioid type of endometrial carcinoma
}

\author{
Shanmugapriya $\mathbf{M}^{1}$, Sudha $\mathbf{M}^{2}$, Geetha $\mathbf{P}^{3}$ \\ ${ }^{1}$ Dr. M. Shanmugapriya, Assistant Professor, Department of Pathology, Meenakshi Medical College and Research \\ Institute, Kanchipuram, Tamil Nadu, India. ${ }^{2}$ Dr. M. Sudha, Assistant Professor, Department of Pathology, \\ Melmaruvathur Adhiparasakthi Institute of Medical Sciences \& Research Institute, Melmaruvathur, Tamil Nadu, India, \\ ${ }^{3}$ Dr. Geetha Prakash, Professor, Department of Pathology, Meenakshi Medical College and Research Institute, \\ Kanchipuram, Tamil Nadu, India.
}

Address for Correspondence: Dr. M. Sudha, Assistant Professor, Department of Pathology, Melmaruvathur Adhiparasakthi Institute of Medical Sciences \& Research Institute, Melmaruvathur, Tamil Nadu, India. Email: dr.sudhasanjnah@gmail.com

\begin{abstract}
Background: To analyze the role of PTEN expression in hyperplastic and neoplastic endometrium by immunohistochemistry and to find the grades of PTEN expression in neoplastic, hyperplasic and normal endometrium. Methods: 100 numbers of endometrial samples were studied retrospectively. The tissue were fixed in formalin and taken up for routine histopathological and immunohistochemistry studies. Results: In our study PTEN is well expressed in a score of 2+ and 1+ in cyclical endometrium and hyperplasia without atypia whereas altered PTEN expression was noted in atypical hyperplasias and endometroid type of endometrial carcinoma in a score of 0and 1+. Conclusion: The current study proved that PTEN expression is down regulated in endometrial pathological condition. Immunohistochemical evaluation of PTEN expression was useful in screening precancerous hyperplasia lesions and detecting earliest stage of endometrial carcinogenesis.
\end{abstract}

Keyword: Phosphatase and tensin homologue (PTEN), Endometrial hyperplasia (EH), Complex atypical hyperplasia $(\mathrm{CAH})$, Endometrioid endometrial carcinoma (EEC).

\section{Introduction}

Endometrial carcinoma is the most common malignancy of the female genital tract and its most frequent subtype is endometrioid type of endometrial carcinoma (Type1 cancers) [1, 2, 3]. Endometrial Carcinoma (Type1) usually develops in perimenopausal women in the setting of hyperestrogenism and frequently coexists with $\mathrm{CAH}$ (EIN). They are typically low-grade tumors without deep myometrial invasion [2, $3,4]$. Despite its prevalence, the molecular mechanisms of endometrial carcinogenesis have been poorly understood. The tumor suppressor gene named PTEN (Phosphatase and Tensin Homologue) also called MMAC1, is located on chromosome 10q23. It is somatically mutated in several types of tumor [2, 3]. PTEN gene appears to be normally regulated, with

Manuscript received: $25^{\text {th }}$ February 2017 Reviewed: $4^{\text {th }}$ March 2017

Author Corrected: $11^{\text {th }}$ March 2017

Accepted for Publication: $18^{\text {th }}$ March 2017 greatest physiological endometrial gland expression in an oestrogen rich environment. Thus the effects of diminished PTEN tumor suppressor function is directly proportional to cancer risk particularly in high estrogenic states [3, 4]. In spite of underlying mechanism, immunohistochemistry study of PTEN expression is an effective screening method for endometrial carcinoma. Endometrial hyperplasia is the ideal benign counterpart for endometrioid carcinoma while atypical hyperplasia is a precursor lesion [5].

Endometrial cancer typically occurs in peri and postmenopausal women, although it may also be seen in premenopausal women, particularly in the setting of hyperestrogenism. Interestingly, studies have also shown that the relationship between PTEN and prognosis in several cancers, including endometrial carcinoma $[2,4,5]$. 


\section{Research Article}

The current study was designed to investigate the normal, hyperplastic, and neoplastic endometrial glands expression by using PTEN as marker and differentiate between hyperplastic and malignant endometrial glands especially in small biopsies, thereby evaluating the role of possibility in early diagnosis of endometrial premalignant lesions.

\section{Materials and Methods}

The study was conducted at Department of Pathology, in Meenakshi Medical College, kanchipuram, Tamilnadu, India. 100 samples of Endometrial specimens, were obtained by hysterectomy and dilatation and curettage (D \& C) from Department of Gynaecology. The tissues were fixed in formalin and taken up for routine histopathological studies and IHC studies. Of the 100 numbers of study cases, 25, 25, 12, 8, 14, 6, and 10 were of proliferative phase, secretory phase, simple hyperplasia without atypia, simple hyperplasia with atypia, complex hyperplasia without atypia, complex hyperplasia with atypia, endometrial carcinoma respectively.

Inclusion criteria: Cases from June 2014 to November 2015 were selected.

Exclusion criteria: Specimens with evidence of chronic non specific endometritis, endometrial polyp, mixed pattern and fibroid are excluded.

Histopathology technique: The specimens were examined grossly, their representative sections were taken and then processed using routine procedure of dehydration, clearing and embedding. The paraffin embedded sections were cut to 4 $\mu \mathrm{m}$ thickness and all were stained with H\&E. Further, $3 \mu \mathrm{m}$ thick sections of the paraffin embedded blocks were taken in Poly L Lysine smeared slides for immunohistochemical (IHC) techniques.

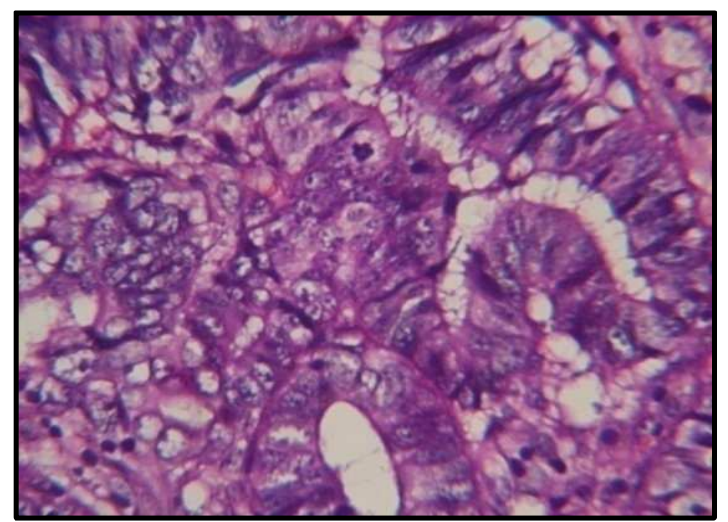

a. Histopathology of Endometrial carcinoma grade $3(\mathrm{H} \& \mathrm{E}, 400 \mathrm{X})$

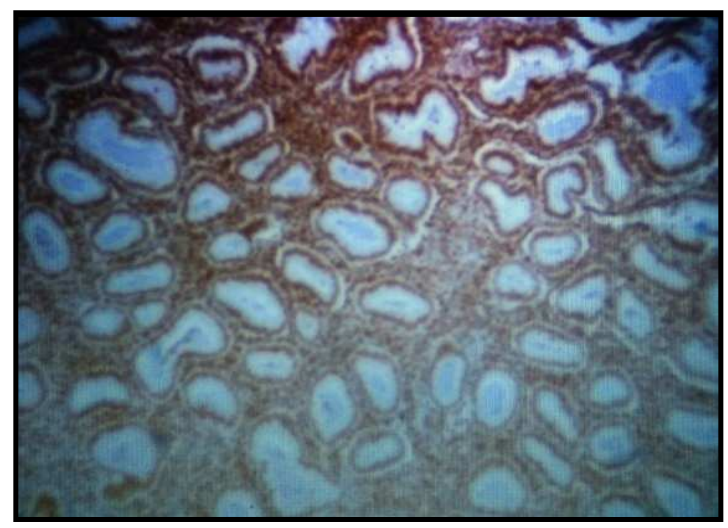

c. Immuno expression PTEN positive in secretory phase of endometrium (IHC, 100x)

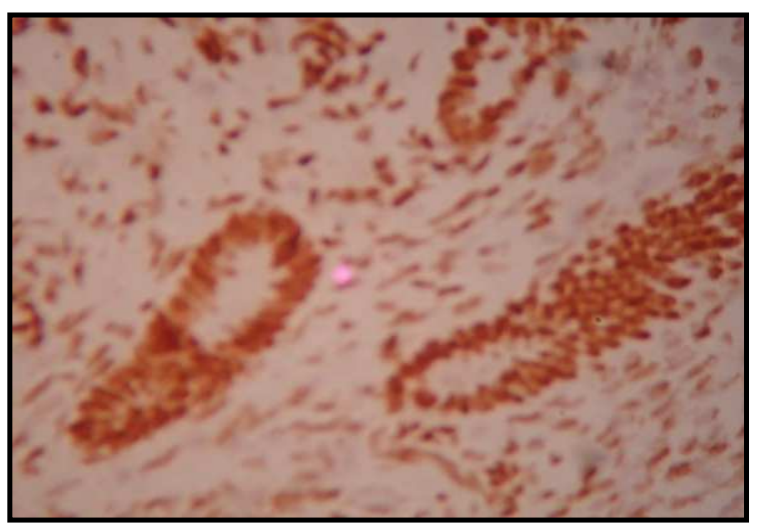

b. Immunohistochemical staining of PTEN antibody strongly positivity in normal proliferative endometrium (IHC, 100X)

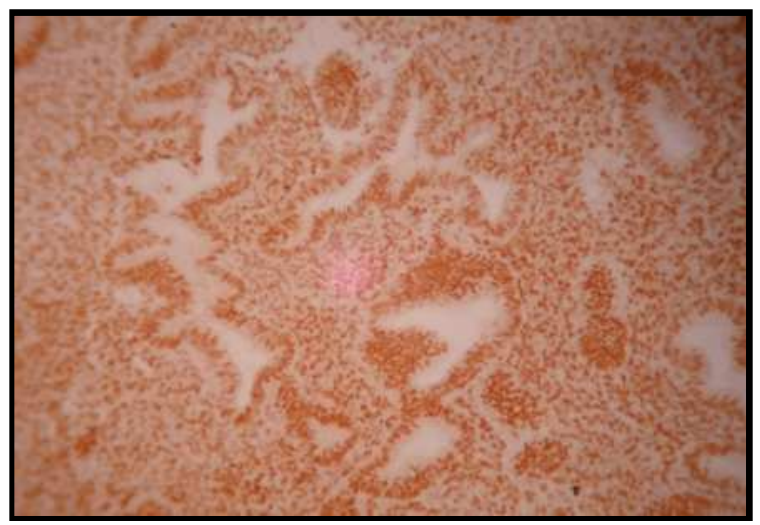

d. Immunohistochemical staining using PTEN antibody showing more than $50 \%$ positive area in simple hyperplasia of endometrium (IHC,100X) 


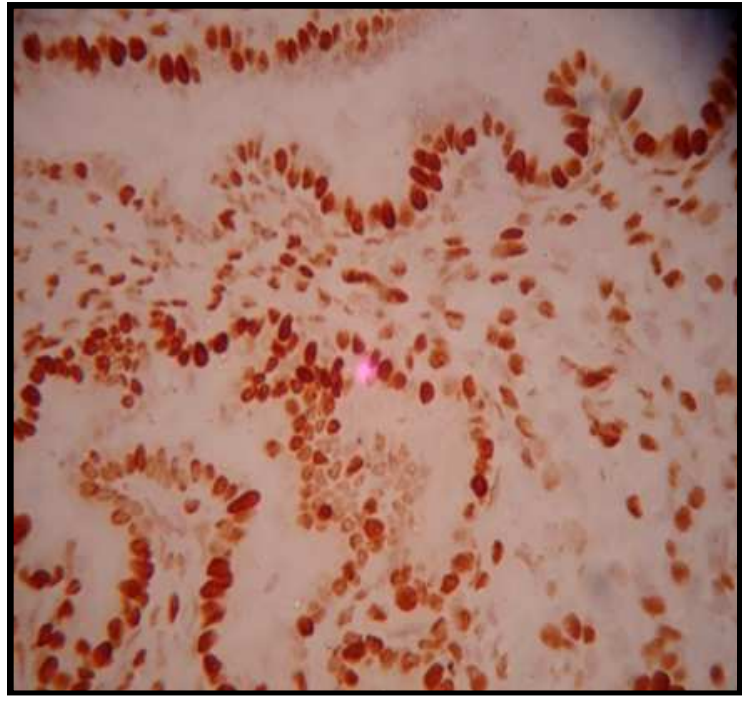

e. Immunohistochemistry of atypical complex hyperplasia of endometrium using PTENantiboby showing weak and focal reaction (IHC,400x)

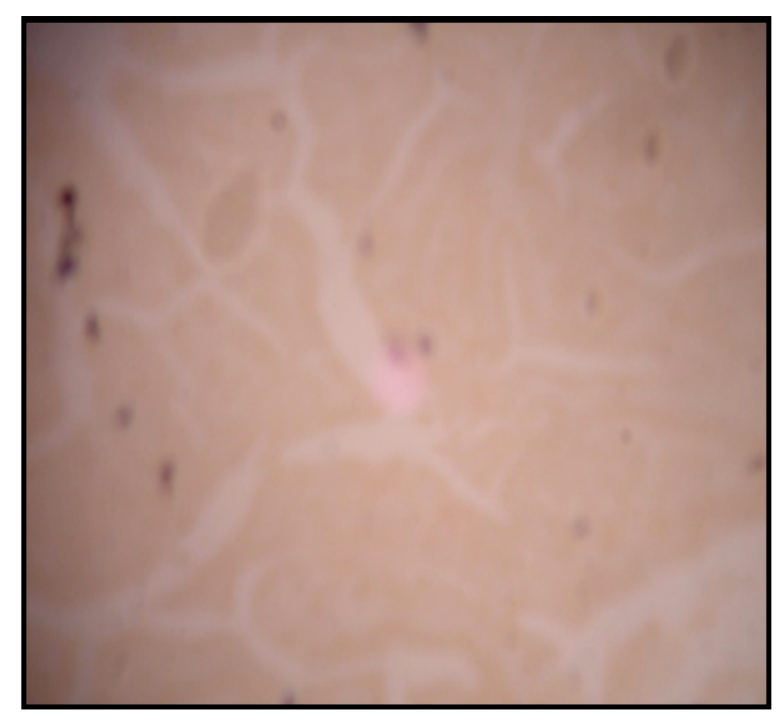

f. PTEN immunochemistry showing complete loss of reaction in endometrioid type of endometrial carcinoma (IHC,100X)

\section{Fig-1: Immunohistochemistry with PTEN antibody}

Immunohistochemistry procedure: The IHC technique for $3 \mu \mathrm{m}$ blocks were performed using the Avidin Biotin Complex (ABC) detection system, with a mouse anti-PTEN monoclonal primary antibody supplied by M/s. Biogenex, USA. Antigen retrieval was carried out using microwave oven. Slides were incubated at $600{ }^{\circ} \mathrm{C}$ overnight. Deparafinisation of sections were done using two changes of xylene 15 minutes each and Hydrated through graded alcohols. Primary blocking was done using 3\% H2O2 in Methanol for 20 minutes and washed in distilled water for 3 minutes. Followed with, two changes of TBST buffer wash of 5 minutes each. Secondary blocking was done using $3 \%$ BSA for 20 minutes. Incubated with primary antibody for $30 \mathrm{~min}$ at room temperature and washed in two changes of TBST buffer for 5 minutes each. Tissue sections were covered with Poly Excel Target Binder and incubated for 10min at room temperature. Washed in TBST buffer 3 changes each for 2 minutes. Tissue sections covered with Poly Excel Poly HRP were incubated for 10 minutes at room temperature. Washed in TBST buffer 3 changes each for 2 minutes.

Sections covered with Stunn DAB chromogen solution were incubated for 5 minutes at room temperature (Solution preparation- Stunn DAB buffer $1 \mathrm{ml}+$ Stunn DAB chromogen 1 drop-mixed well and stored in the dark, stable for a week at 2-80 ${ }^{\circ} \mathrm{C}$ ). Washed in TBST buffer 2 changes each for 5 minutes and counter stained with Harri's Haematoxylin for 10 minutes at room temperature. Sections were dehydrated using graded alcohols. Clearing was done with 2 changes of Xylene each for 5 minutes, the respective sections were closed with mounting medium DPX. According to Soheila sarmadi et al [1] and Kapucuoglu et al [5] immunoreactivity of PTEN was regarded as positive when brown staining was localized in the nuclei or cytoplasm of glandular cells for each specimen. Staining of cells was scored as negative if < $10 \%, 1+$ if $10 \%-50 \%$ and $2+$ if $>50 \%$ area of slide was stained positive. Immunohistochemistry microscopic images of proliferative, secretory, simple hyperplasia, atypical complex hyperplasia, and endometrioid type of endometrial carcinoma are shown in Fig 1.

\section{Results}

Based on the percentage of staining area IHC results are graded from 0 to $2+$. Less than $10 \%$ staining area taken as negative, $10 \%$ to $50 \%$ is taken as $1+$ and $>50 \%$ is taken as $2+$. The PTEN results of the samples investigated are given in Table 1 . In simple hyperplasia without atypia only $10 \%$ showed negativity whereas, in simple hyperplasia with atypia, $40 \%$ cases showed negativity. In complex hyperplasia PTEN negativity is seen in $22 \%$ of cases without atypia and $50 \%$ of cases with atypia. It shows PTEN mutation is seen in hyperplasia with atypia. The difference of expression between simple and complex hyperplasia with and without atypia is statistically significant [Fig 2]. 
Research Article

\section{Table-1: PTEN Result.}

\begin{tabular}{|c|c|c|c|c|}
\hline & Negative & $\mathbf{1 +}$ & $\mathbf{2 +}$ & Total \\
\hline Proliferative phase & 0 & 1 & 24 & 25 \\
\hline Secretory phase & 0 & 4 & 21 & 25 \\
\hline Simple Hyperplasia without atypia & 1 & 3 & 8 & 12 \\
\hline Simple Hyperplasia with atypia & 3 & 2 & 3 & 8 \\
\hline Complex Hyperplasia without atypia & 2 & 7 & 5 & 14 \\
\hline Complex Hyperplasia with atypia & 4 & 1 & 1 & 6 \\
\hline Endometrioid Endometrial carcinoma & 7 & 3 & 0 & 10 \\
\hline
\end{tabular}

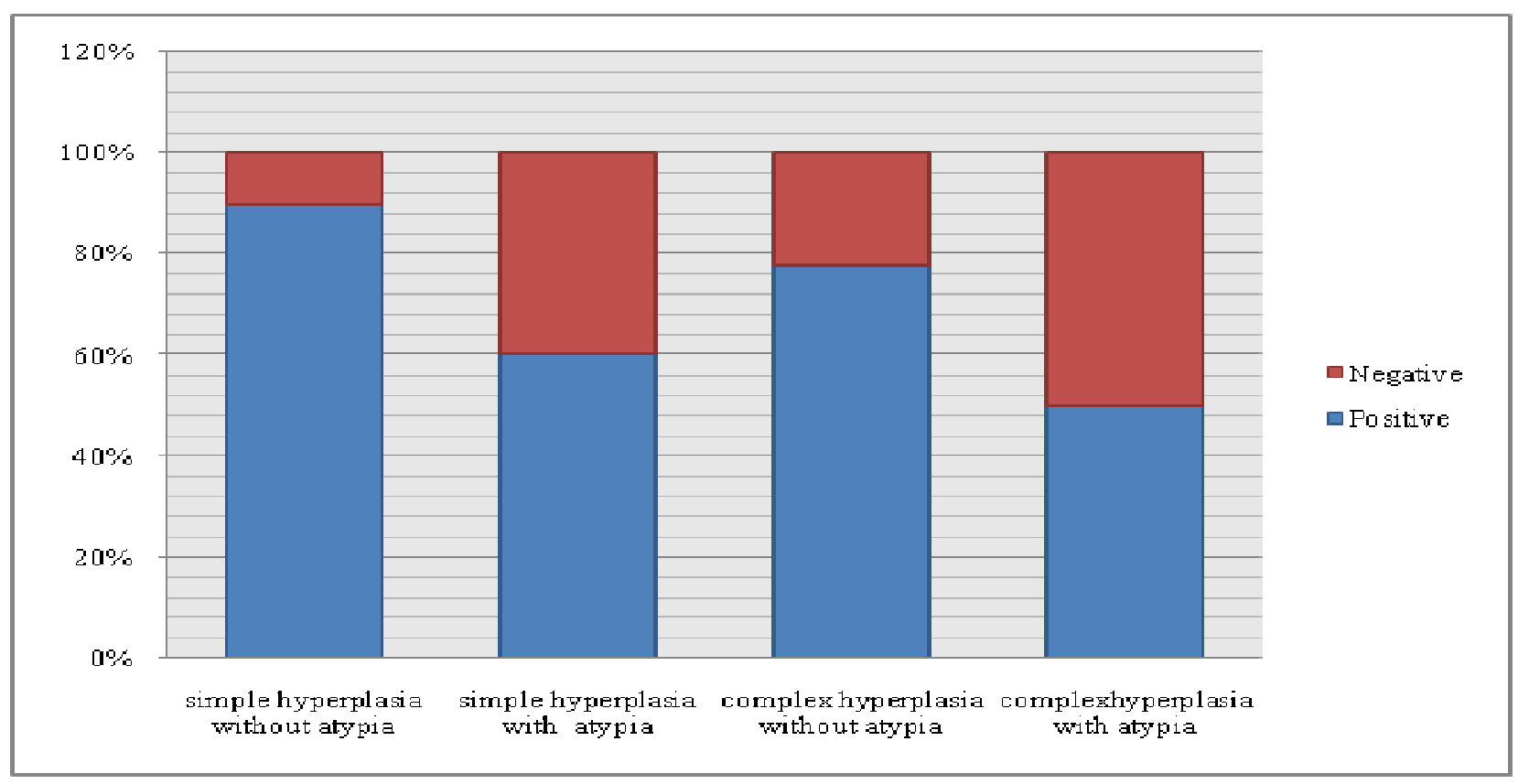

Fig-2: PTEN in Endometrial Hyperplasia

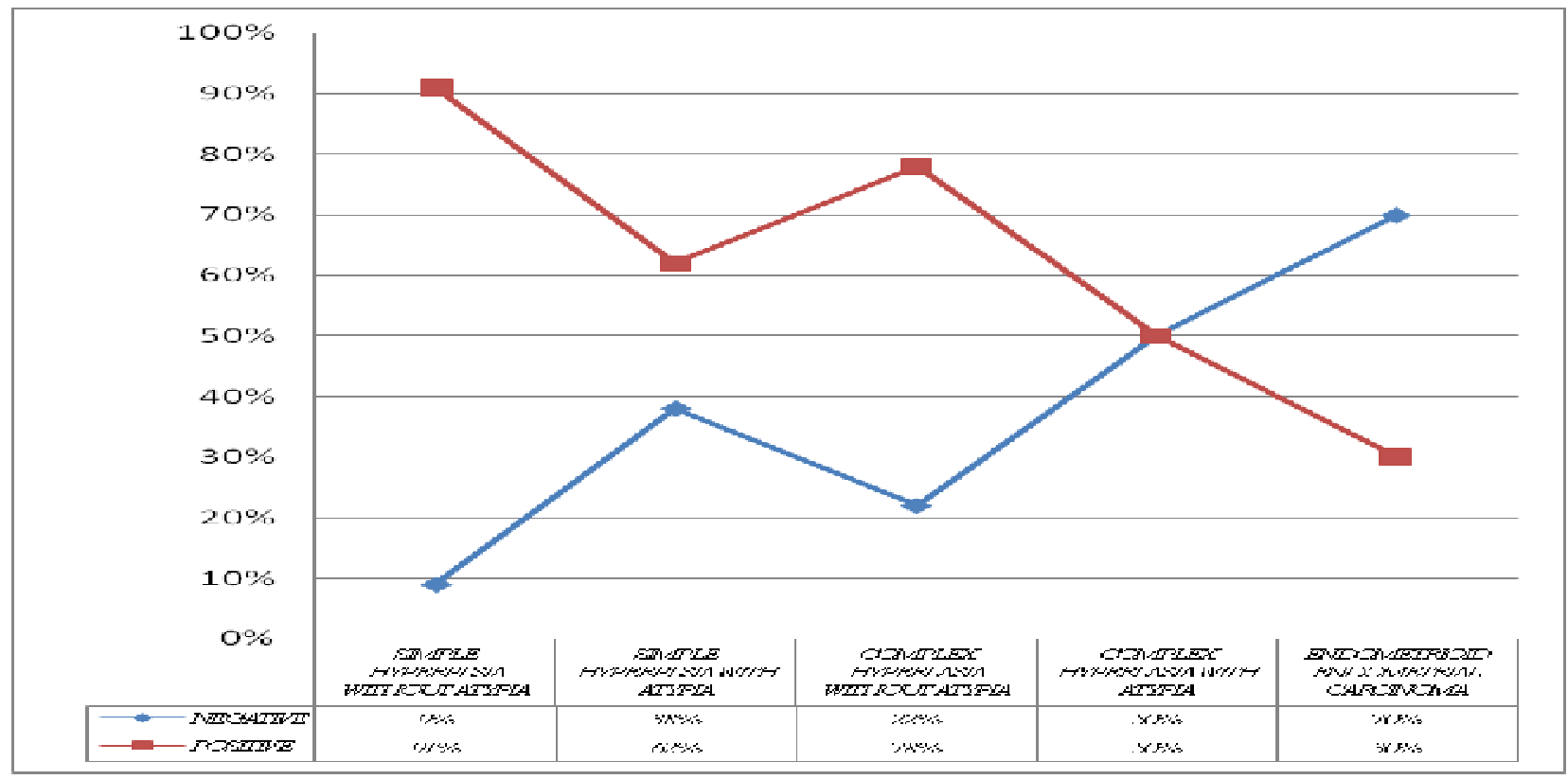

Fig-3: PTEN in Hyperplasia Vs Carcinoma 
Research Article

PTEN is expressed above $90 \%$ of cases in simple hyperplasia without atypia, $62 \%$ of cases in simple hyperplasia with atypia, above $70 \%$ in complex hyperplasia without atypia and only $50 \%$ in complex hyperplasia with atypia, in endometrial carcinoma only $30 \%$ of cases showed positivity. There is a down regulation of PTEN expression from hyperplasia with atypical changes to carcinoma. In endometrioid endometrial carcinoma PTEN is negative in $70 \%$ of cases and it is positive in $30 \%$ cases [Fig 3].

\section{Discussion}

The term "endometrial neoplasia" is a spectrum of morphologic alterations that range from endometrial hyperplasia to the different varieties of endometrial carcinoma [2,4]. Hyperplasia and carcinoma represent only two different points along the spectrum of endometrial proliferations. Endometrial hyperplasia is defined by the 2003 World Health Organization (WHO) classification as a spectrum of morphologic alterations ranging from benign changes to premalignant disease, caused by an abnormal hormonal environment $[6,7]$.

A group of investigators has proposed an alternative term "endometrial intraepithelial neoplasia (EIN)" to define premalignant endometrial lesions that show specific morphologic features and share molecular alterations with endometrial carcinoma [6,7].

It has been difficult to determine the malignant potential of the various types of endometrial hyperplasia, due to problems in terminology and lack of follow-up data. Microsatellite instability, mutations of PTEN and K-ras, and nuclear accumulation of $\beta$-catenin are the most characteristic molecular alterations associated with these tumors $[4,7,8]$.

PTEN is strongly immunoreactive in all cases of proliferative and secretory phase in the current study. These findings agreed with Soheila Sarmadi et al (2009), in his study, PTEN immunoreactivity was noted in all normal proliferative endometrium $(29 / 29,100 \%)$. PTEN expression was significantly higher in cyclical endometrium than in atypical hyperplasia and endometrioid carcinoma [1].

In the present study PTEN is well expressed in a score of $1+, 2+$ in simple hyperplasia without atypia $90 \%$ cases and complex hyperplasia without atypia $78 \%$. Only $10 \%$ to $20 \%$ are negatively expressed.

There is no much difference in pattern of expression between normal and simple hyperplasia. In a study by Hongbo Sun et al, out of 24 cases of simple hyperplasia only one case is mutated and in a follow up study of all cases $8 \%$ of simple hyperplasia with atypia progressed to carcinoma [7]. Its difference is statistically insignificant. This finding is slightly lower to study by Soheila Sarmadi et al (2009) who reported 100\% positivity in simple hyperplasia [1].

In the current study PTEN expression in simple hyperplasia with atypia is $60 \%$, complex hyperplasia with atypia is $50 \%$. In atypical hyperplasia $40 \%$ to $50 \%$ of cases are negatively expressed. In a study by Soheila Sarmadi et al (2009) 25\% negativity in atypical complex hyperplasia was reported [1]. Levine et al and Maxwell et al [8] found complete loss of PTEN in 20\% of atypical complex samples. Kapucuogluet al [5] also found complete loss of PTEN in 20\% of atypical complex samples. In a current study loss of PTEN expression is seen in $70 \%$ cases of EECA. Out of 10 cases of endometrioid endometrial carcinoma 7 cases showed PTEN negativity.In a study by Soheila Sarmadi et al (2009), the lowest PTEN immunoreactivity was detected in Endometrioid endometrial carcinoma and deteted loss of PTEN expression in 52\% of Endometrioid endometrial carcinoma and $25 \%$ of Atypical complex hyperplasia [1]. Orbo et al [9] reported loss of PTEN protein expression in 55\% of specimens in patients with subsequent endometrioid endometrial carcinoma.

In a study by Yasunobu Kanamori et al (2002) out of 98 advanced cases, 64 (65.3\%) showed negative or mixed PTEN staining; their survival rate was significantly lower than that of PTEN-positive cases [10]. In a study by Erkanli $\mathrm{S}$ et al loss of PTEN is found in $80 \%$ of endometrial carcinomas [11]. In a study by $\mathrm{N}$ Terakawa et al, out of 98 advanced cases, 64 cases $(65 \%)$ showed negative or heterogeneous PTEN staining; their survival rate was significantly lower than that of PTEN-positive cases [2]. Gema Moreno-Bueno et al (2004) reported 50\% positivity in endometrioid endometrial carcinoma [12].

Mutter GL et al (2000) found, Altered PTEN expression as a diagnostic marker for the earliest endometrial precancers [13]. Latta $\mathrm{E}$ et al (2002) found mutations of the PTEN tumour suppressor gene have been identified 


\section{Research Article}

in histologically normal-appearing endometrium exposed to oestrogen, $18-55 \%$ of endometrial precancers and $26-80 \%$ of EECs [14].

Therefore in our study, PTEN negative immunereactivity was detected in the majority of Endometrioid endometrial carcinoma and atypical hyperplasia but none in secretory and proliferative endometrium. PTEN is downregulated in endometrioid type of endometrial carcinoma and combination of PTEN expression and histological features have greatest diagnostic utility in endometrial hyperplasia and carcinoma.

\section{Conclusion}

PTEN is well expressed in cyclical endometrium of both proliferative and secretory phase and showed altered expression in hyperplasia with atypia compared with hyperplasia without atypia. In Endometrioid endometrial carcinoma, PTEN is negatively expressed in almost $70 \%$ of cases, so it indicates that downregulation of PTEN in atypical hyperplasia and endometrioid endometrial carcinoma. So we felt that PTEN expression is partly associated with endometrial cancers through a premalignant phase and decreased PTEN expression tended to associate with malignant features of endometrium with a significant statistical difference of PTEN immunoreactivity between group of normal, hyperplasia and carcinoma.

Funding: Nil, Conflict of interest: None initiated, Permission from IRB: Yes

\section{References}

1.Sarmadi S, Izadi-Mood N, Sotoudeh K, Tavangar SM. Altered PTEN expression; a diagnostic marker for differentiating normal, hyperplastic and neoplastic endometrium. Diagnostic pathology. 2009 Nov 25;4 (1):41.

2.Terakawa N, Kanamori Y, Yoshida S. Loss of PTEN expression followed by Akt phosphorylation is a poor prognostic factor for patients with endometrial cancer. Endocrine-related cancer. 2003 Jun 1;10(2):203-8.

3. Chow LM, Baker SJ. PTEN function in normal and neoplastic growth. Cancer letters. 2006 Sep 28;241 (2):184-96.

4. Risinger JI, Hayes AK, Berchuck A, Barrett JC. PTEN/MMAC1 mutations in endometrial cancers. Cancer research. 1997 Nov 1;57(21):4736-8.
5. Kapucuoglu N, Aktepe F, Kaya H, Bircan S, Karahan $\mathrm{N}$, Çiriş M. Immunohistochemical expression of PTEN in normal, hyperplastic and malignant endometrium and its correlation with hormone receptors, bcl-2, bax, and apoptotic index. Pathology-Research and Practice. 2007 Mar 28;203(3):153-62.

6. Li XC, Song WJ. Endometrial Intraepithelial Neoplasia (EIN) in endometrial biopsy specimens categorized by the 1994 World Health Organization classification for endometrial hyperplasia. Asian Pacific Journal of Cancer Prevention. 2013;14(10):5935-9.

7. Sun H, Enomoto T, Fujita M, Wada H, Yoshino K, Ozaki K, Nakamura T, Murata Y. Mutational analysis of the PTEN gene in endometrial carcinoma and hyperplasia. American journal of clinical pathology. 2001 Jan 1;115(1):32-8.

8. Maxwell GL, Risinger JI, Gumbs C, Shaw H, Bentley RC, Barrett JC, Berchuck A, Futreal PA. Mutation of the PTEN tumor suppressor gene in endometrial hyperplasias. Cancer research. 1998 Jun $15 ; 58(12): 2500-3$.

9. Ørbo A, Nilsen MN, Arnes MS, Pettersen I, Larsen K. Loss of expression of MLH1, MSH2, MSH6, and PTEN related to endometrial cancer in 68 patients with endometrial hyperplasia. International journal of gynecological pathology. 2003 Apr 1;22(2):141-8.

10. Kanamori Y, Kigawa J, Itamochi H, Sultana H, Suzuki M, Ohwada M, Kamura T, Sugiyama T, Kikuchi Y, Kita T, Fujiwara K. PTEN expression is associated with prognosis for patients with advanced endometrial carcinoma undergoing postoperative chemotherapy. International journal of cancer. 2002 Aug 20;100 (6):686-9.

11. Erkanli S, Kayaselcuk F, Kuscu E, Bagis T, Bolat F, Haberal A, Demirhan B. Expression of survivin, PTEN and p27 in normal, hyperplastic, and carcinomatous endometrium. International Journal of Gynecological Cancer. 2006 May 1;16(3):1412-8.

12. Moreno- Bueno G, Rodríguez-Perales S, Sánchez-Estévez C, Marcos R, Hardisson D, Cigudosa JC, Palacios J. Molecular alterations associated with cyclin D1 overexpression in endometrial cancer. International journal of cancer. 2004 Jun 10;110 (2): 194-200. 
13. Mutter GL, Lin MC, Fitzgerald JT, Kum JB, Baak JP, Lees JA, Weng LP, Eng C. Altered PTEN expression as a diagnostic marker for the earliest endometrial precancers. Journal of the National Cancer Institute. 2000 Jun 7;92(11):924-30.
14. Latta E, Chapman WB. PTEN mutations and evolving concepts in endometrial neoplasia. Current Opinion in Obstetrics and Gynecology. 2002 Feb 1;14(1):59-65.

\section{How to cite this article?}

Shanmugapriya M, Sudha M, Geetha P. A study of PTEN expression in endometrial hyperplasia and endometrioid type of endometrial carcinoma. Trop J Path Micro 2017;3(1):39-45.doi: 10.17511/jopm.2017.i1.07. 